Polymer Journal, Vol. 39, No. 4, pp. 304-317 (2007)

(C) 2007 The Society of Polymer Science, Japan

\title{
Kinetics of Anionic Polymerization of Polybutadienyl Lithium in Benzene: An Osmotic Effect on Propagation Process
}

\author{
Yohei Oishi, Yumi MatsumiYa, and Hiroshi WatanabE ${ }^{\dagger}$ \\ Institute for Chemical Research, Kyoto University, Uji, Kyoto 611-0011, Japan
}

(Received November 2, 2006; Accepted December 27, 2006; Published February 26, 2007)

\begin{abstract}
The kinetics of the polymerization (propagation) process of protonated polybutadienyllithium (hPB) in a nonpolar solvent, deuterated benzene (dBz), was examined with ${ }^{1} \mathrm{H}$ NMR. An oligomeric, deuterated butadienyllithium (oBLi) was utilized as an initiator in order to avoid a contamination of the initiation process to the NMR data. The hPBLi chains mostly formed aggregate with an average aggregation number $f \cong 4$ through Li at their ends. The residual monomer fraction $\phi(t)$ did not rigorously exhibit the single-exponential decay with time $t$ expected for the conventionally considered propagation through the dissociated chains, $\phi(t)=\exp (-t / \tau)$ with $\tau=\left\{f / K_{\mathrm{d}}\left[\mathrm{I}_{0}\right\}^{1 / f} / k_{\mathrm{p}}\left(K_{\mathrm{d}}=\right.\right.$ association/dissociation equilibrium constant, $k_{\mathrm{p}}=$ propagation rate constant, $[\mathrm{I}]_{0}=$ molar concentration of initiator at time 0). This deviation from the conventional behavior appeared to be due to competing propagation mechanism through the transiently fused aggregates (suggested from the ${ }^{7} \mathrm{Li}$ NMR data): This fusion-aided propagation should have been osmotically suppressed on an increase of the hPB concentration (decrease of $\phi$ ) to give the deviation. The propagation was found to be also retarded in the presence of chemically inert deuterated polybutadiene (dPB) chains that just tuned the osmotic environment for the aggregates, lending support to the molecular idea of the osmotic effect on the propagation. Furthermore, the $\phi(t)$ data in the absence/presence of the dPB chains were semi-quantitatively described by a simple model considering the competition of the propagation mechanisms through the fused $2 f$-mer aggregates and dissociated chains, with the former mechanism vanishing in the late stage of propagation. These results suggested a non-negligible contribution of the fused aggregates to the polymerization kinetics in particular in the early stage. [doi:10.1295/polymj.PJ2006146]

KEY WORDS Anionic Polymerization Kinetics / Polybutadienyl Lithium / Aggregates / Osmotic Effect /
\end{abstract}

The kinetics of living anionic polymerization is an important subject in the field of polymer synthesis. In particular, the polymerization reaction utilizing organolithium (RLi) compounds as the initiator in nonpolar solvents offers a route of synthesizing polydienes (rubbers) of well-controlled microstructures and thus its kinetics has been extensively studied. ${ }^{1-10}$ These studies indicated that the initiation process of this reaction does not obey the simplest kinetics (RLi + $\mathrm{M} \rightarrow \mathrm{RM}^{-} \mathrm{Li}^{+} ; \mathrm{M}=$ monomer) for which the initiation rate is proportional to the initiator concentration. This deviation has been related to the association of the initiator RLi molecules in nonpolar solvents, although a role this association in the initiation process has not been fully elucidated. ${ }^{4,9,10}$

On the other hand, for the propagation process of the anionic polymerization after full consumption of the RLi molecules, a simple kinetics shown in Scheme 1 seems to have been accepted. In this kinetics, the anionic polymer chains, PLi, are in chemical equilibrium with their star-like $f$-mer aggregates $(\mathrm{PLi})_{f}$ bound at the Li ends (as detected from scattering experiments ${ }^{10-16}$ ), and the propagation occurs only through the dissociated PLi chains because the aggre-

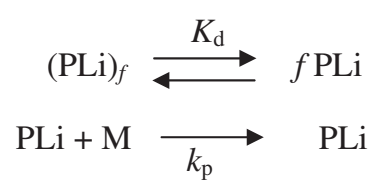

Scheme 1. Widely accepted route of propagation through dissociated chain.

gates are considered to be well stabilized and thus inert for the propagation. The time $(t)$ evolution of the molar concentration of the monomer, $[\mathrm{M}]$, is then described by

$$
\frac{\mathrm{d}[\mathrm{M}]}{\mathrm{d} t}=-k_{\mathrm{p}}[\mathrm{P}][\mathrm{M}]
$$

with $k_{\mathrm{p}}$ and $[\mathrm{P}]$ being the propagation rate constant and the molar concentration of the dissociated PLi chains, respectively. Furthermore, in most cases, the chains are considered to be predominantly in the aggregated state. Then, the dissociated chain concentration $[\mathrm{P}]$ is specified by

$$
K_{\mathrm{d}}=\frac{[\mathrm{P}]^{f}}{\left[\mathrm{P}_{f}\right]} \cong \frac{[\mathrm{P}]^{f}}{\left\{[\mathrm{I}]_{0} / f\right\}}
$$


Here, $\left[\mathrm{P}_{f}\right]$ and $K_{\mathrm{d}}$ are the molar concentration of the $(\mathrm{PLi})_{f}$ aggregates and the equilibrium constant between the dissociated and aggregated chains ( $c f$. Scheme 1), respectively, and $[\mathrm{I}]_{0}$ is the molar concentration of the initiator just before the propagation process.

Equations 1 and 2 lead to a single-exponential decay of the residual fraction of the monomer,

$$
\phi(t) \equiv \frac{[\mathrm{M}]}{[\mathrm{M}]_{0}}=\exp \left\{-k_{\mathrm{p}}\left(\frac{K_{\mathrm{d}}[\mathrm{I}]_{0}}{f}\right)^{1 / f} t\right\}
$$

Here, $[\mathrm{M}]_{0}$ is the monomer concentration at the onset of the propagation (just after consumption of the initiator, $\mathrm{RLi}$ ), and $t$ is a time after this onset. This type of single-exponential decay should be observed irrespective of the details of the initiation step and a distribution of the aggregation number $f$, as long as all aggregates are inert for the propagation step. Indeed, most of the kinetic experiments ${ }^{1-8}$ lent support to the single-exponential decay (though in limited ranges of time, as explained later in more details).

A recent experiment ${ }^{16}$ for living polybutadienyllithium (PBLi) chains poses a delicate question related to this chemical inertness of the aggregates. For monodisperse PBLi chains of the molecular weights $M=$ $2.6 \times 10^{3}-9.6 \times 10^{3}$ and the mass concentrations $C=$ $0.016-0.096 \mathrm{~g} \mathrm{~cm}^{-3}$ fully polymerized in benzene (i.e., after completion of the propagation process), light/neutron scattering experiments ${ }^{15,16}$ indicated that the PBLi chains predominantly form the $(\mathrm{PBLi})_{f}$ aggregates with the average aggregation number $f \cong 4$ (and a very minor fraction of huge aggregates with $f \gg 4$ ), and a ${ }^{7} \mathrm{Li}$ NMR experiment ${ }^{16}$ revealed that several different $\mathrm{Li}$ species coexist in the system and a thermal exchange among these species has a $C$ and $M$-dependent characteristic time $\tau_{\text {ex }}(=0.1-10 \mathrm{~s}$ at room temperature). Furthermore, this $\tau_{\mathrm{ex}}$ was associated with an activation energy, $\Delta E=88 \mathrm{~kJ} \mathrm{~mol}^{-1}$, that was considerably lower than the energy $\Delta E_{\mathrm{C}-\mathrm{Li}}$ required for cleaving a C-Li bond ${ }^{17}\left(>150 \mathrm{~kJ} \mathrm{~mol}^{-1}\right)$. The $C$ - and $M$-dependencies of $\tau_{\text {ex }}$ as well as this $\Delta E$ value suggest that the $\mathrm{Li}-\mathrm{Li}$ exchange occurs mainly through a thermal fusion of the $f$-mer aggregates into a larger aggregate, not through an independent dissociation of respective PBLi chains from the $f$-mer aggregates. (If this independent dissociation is the main, rate-determining route for the $\mathrm{Li}-\mathrm{Li}$ exchange, $\tau_{\mathrm{ex}}$ should have exhibited no $C$ - and $M$-dependencies and its activation energy should have agreed with $\left.\Delta E_{\mathrm{C}-\mathrm{Li}}\right)$

The transiently fused aggregate should be less stable, i.e., more reactive compared to the smaller $f$ mer aggregates ( $f=4$ for PBLi in benzene). Thus,

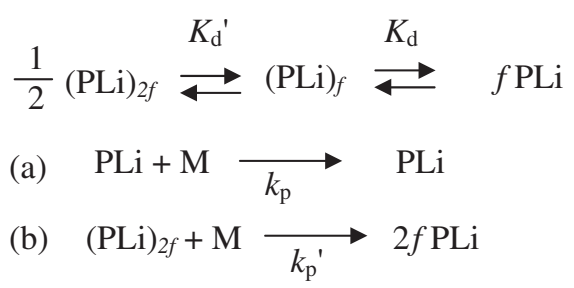

Scheme 2. Competitive propagation routes through (a) dissociated chains and (b) fused $2 f$-mer aggregates.

in the presence of the monomers (which was not the case for the above scattering/NMR experiments), the fused aggregate may work as an active species for the propagation step in addition to the dissociated PBLi chain. In relation to this point, quantum calculations suggested that the propagation through the independently dissociated single chain is hardly probable. ${ }^{17,18}$ The simplest propagation route through the transient $2 f$-mer aggregates is shown in Scheme 2. (The coexisting huge aggregates with $f \gg 4$ has a very minor fraction in the system and their contribution to the propagation process can be neglected. ${ }^{15}$ )

If this additional route contributes to the propagation process, no single-exponential decay is expected for the residual fraction of the monomer, $\phi(t)=$ $[\mathrm{M}] /[\mathrm{M}]_{0}$. This expectation is not necessarily contradicting to the single-exponential decay seen in the previous studies for the polymerization of dienes, ${ }^{1-8}$ because most of those studies traced the decay of $\phi(t)$ only down to $\phi(t)=0.1-0.2$ (to 0.05 at the lowest ${ }^{8}$ ): The fusion-aided mechanism should contribute to the propagation process less significantly with decreasing $\phi(t)$ because the corresponding increases of $M$ and $C$ in the polymerizing system enhance the osmotic barrier for the fusion, but this change of the contribution is not necessarily resolved well in the limited ranges of $\phi(t)$.

Thus, we have made ${ }^{1} \mathrm{H}$ and ${ }^{7} \mathrm{Li}$ NMR measurements to examine the hypothesis of fusion-aided propagation in $\mathrm{PBLi} / \mathrm{Bz}$ anionic polymerization systems. A deuterated butadienyllithium oligomer was utilized to start the propagation of the protonated $\mathrm{B}$ monomer, which allowed the ${ }^{1} \mathrm{H}$ NMR experiment to accurately trace the decay of $\phi(t)$ in the purely propagation process down to $\phi(t)=0.01$ (or even smaller). The exchange of the Li species of the polymerizing PBLi chains was monitored with ${ }^{7} \mathrm{Li}$ NMR. These experiments revealed that $\phi(t)$ does not decay in a rigorously single-exponential fashion and this behavior is in harmony with the Li-Li exchange, as expected for the fusion-aided propagation mechanism having a nonnegligible contribution in the early stage of the propagation process. Furthermore, we also examined the decay of $\phi(t)$ in the presence of non-anionic, deuterated $\mathrm{PB}$ chains that just tuned the osmotic environment 
for the aggregates of living PBLi. This type of experiment, never made in previous studies, revealed a retarded decay of $\phi(t)$ due to the dPB chains being consistent with the fusion-aided propagation mechanism. This paper presents details of these results.

\section{EXPERIMENTAL}

\section{Materials and Sample Preparation}

Protonated and deuterated butadiene monomers (hB and $\mathrm{dB}$; purchased from Tokyo Kasei and CDN Isotopes, respectively), deuterated benzene $(\mathrm{dBz}$; Aldrich; proton content $=0.5 \%$ ), heptane (Wako), sec-butyl chloride (Aldrich), and methanol (Wako) were purified with the standard methods. ${ }^{1}$ All chemical reactions were made in glass ampoules/flasks with the aid of standard high vacuum operation using constrictions and breakable seals.

$s e c$-Butyllithium ( $s$-BuLi) was synthesized from $\mathrm{Li}$ metal (Aldrich) and sec-butyl chloride in heptane. Oligomeric butadienyl lithium (oBLi) was synthesized from $\mathrm{dB}$ monomer and $s$-BuLi in $\mathrm{dBz}$ at $40^{\circ} \mathrm{C}$, and its characteristics $\left(M_{\mathrm{w}}=6.2 \times 10^{2}, M_{\mathrm{w}} / M_{\mathrm{n}}=1.06\right)$ were determined with GPC.

Three batches of deuterated polybutadiene (dPB), utilized as an additive that tuned the osmotic environment during the polymerization process, were anionically synthesized with this oBLi initiator and $\mathrm{dB}$ monomer in $\mathrm{dBz}$ at room temperature. The microstructure of PBLi synthesized in this condition is cis:trans:vinyl $\cong 40: 50: 10 .{ }^{1,3}$ The resulting $\mathrm{dPBLi}$ anions in $\mathrm{dBz}$ were terminated/precipitated in large excess of methanol and the supernatant methanol phase (containing lithium methoxide and $\mathrm{dBz}$, the former resulting from the termination reaction) was removed by decantation. The remaining dPB samples were dissolved in $\mathrm{dBz}$ and then precipitated again in excess methanol, and the supernatant was removed by decantation. After this dissolution/precipitation procedure repeated for three times in vacuum, the dPB samples were thoroughly dried with the aid of a high vacuum line and finally dissolved in $\mathrm{dBz}$ and split in several ampoules. The molecular characteristics of the dPB samples determined from GPC were $M_{\mathrm{w}}=15.5 \times 10^{3}$ and $M_{\mathrm{w}} / M_{\mathrm{n}}=1.03$ for a lower- $M$ sample $(\mathrm{dPB}-1), M_{\mathrm{w}}=48.7 \times 10^{3}$ and $M_{\mathrm{w}} / M_{\mathrm{n}}=$ 1.05 for a middle- $M$ sample (dPB-2) and $M_{\mathrm{w}}=$ $60.0 \times 10^{3}$ and $M_{\mathrm{w}} / M_{\mathrm{n}}=1.05$ for a higher- $M$ sample (dPB-3).

The materials subjected to the NMR measurements were the anionically polymerizing $\mathrm{hB} / \mathrm{oBLi} / \mathrm{dBz}$ solutions sealed in NMR test tubes made of glass (diameter $=5 \mathrm{~mm}$ ). Some solutions also contained chemically inert $\mathrm{dPB}$ (prepared as above) that tuned the osmotic environment for the propagating hPBLi

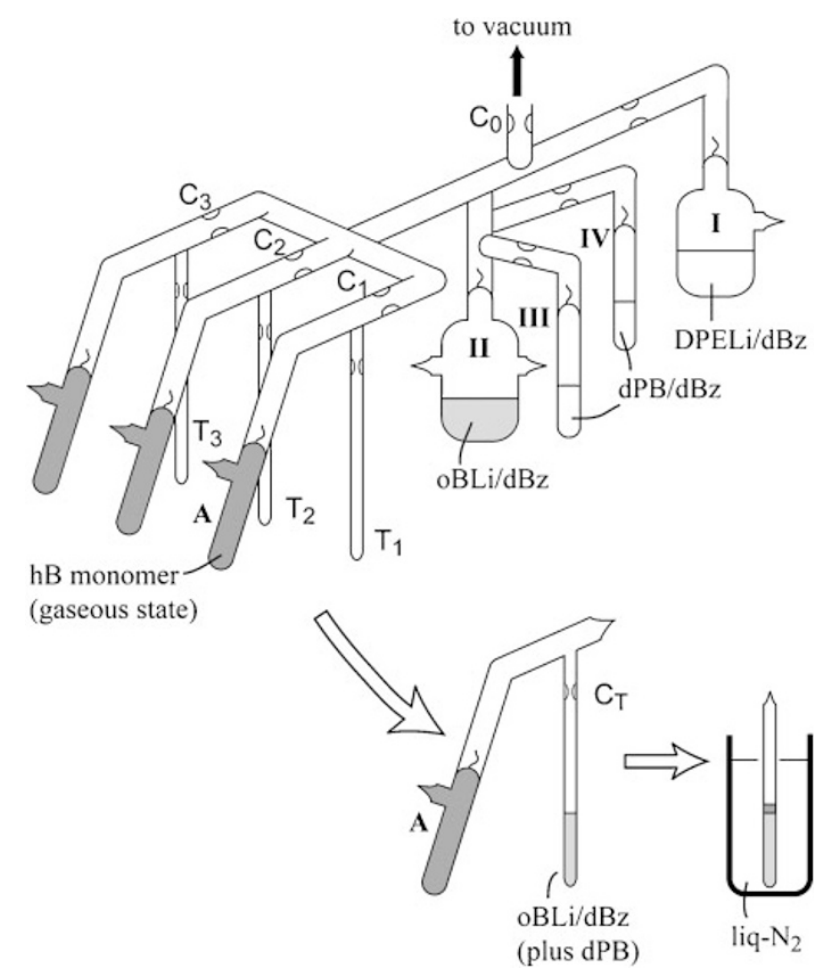

Figure 1. Schematic illustration of a glass apparatus utilized for preparation of $\mathrm{hB} / \mathrm{oBLi} / \mathrm{dBz}$ and $\mathrm{hB} / \mathrm{oBLi} / \mathrm{dPB} / \mathrm{dBz}$ solutions sealed in NMR test tubes. For simplicity of figure, magnetic bars utilized for opening the breakable seals are not shown.

chains. A glass apparatus utilized for sealing these solutions in the NMR tubes is schematically shown in Figure 1. For simplicity of Figure, magnetic bars utilized to open the breakable seals are not shown.

After full evacuation, the apparatus was sealed at the constriction $\mathrm{C}_{0}$ and its inside wall was thoroughly rinsed with a solution of diphenylethylene anion made with $s$-butyllithium (DPELi; $\mathrm{CH}_{3} \mathrm{CH}_{2} \mathrm{CH}\left(\mathrm{CH}_{3}\right) \mathrm{CH}_{2} \mathrm{C}$ $\left.\left(\mathrm{C}_{6} \mathrm{H}_{5}\right)_{2} \mathrm{Li}\right)$ ) in $\mathrm{dBz}$ (separately prepared and sealed in the ampoule I). This DPELi solution was recovered in this ampoule and sealed off. Then, the solution of oBLi (deuterated butadienyllithum oligomer) in $\mathrm{dBz}$ was poured from the ampoule II into one of the NMR tube, $T_{1}$. After sealing off the stem carrying $T_{1}$ at the constriction $\mathrm{C}_{1}$, the $\mathrm{dPB} / \mathrm{Bz}$ solution sealed in the ampoule III was poured into the ampoule II, and a prescribed volume of $\mathrm{dBz}$ (= volume of the $\mathrm{dPB} / \mathrm{Bz}$ solution originally in the ampoule III) was distilled back to the ampoule III in order to minimize a change in the molar concentration of oBLi in the ampoule II. After sealing off the ampoule III, the oBLi/dPB/Bz solution in the ampoule II was poured into the NMR tube $T_{2}$ and the stem carrying $T_{2}$ was sealed off at the constriction $\mathrm{C}_{2}$. For one batch of the polymerization experiment, the other ampoule IV containing the $\mathrm{dPB} / \mathrm{Bz}$ solution was attached to the splitting apparatus, and a similar operation was repeated for this ampoule to seal the $\mathrm{oBLi} / \mathrm{dPB} / \mathrm{Bz}$ solution of a higher 


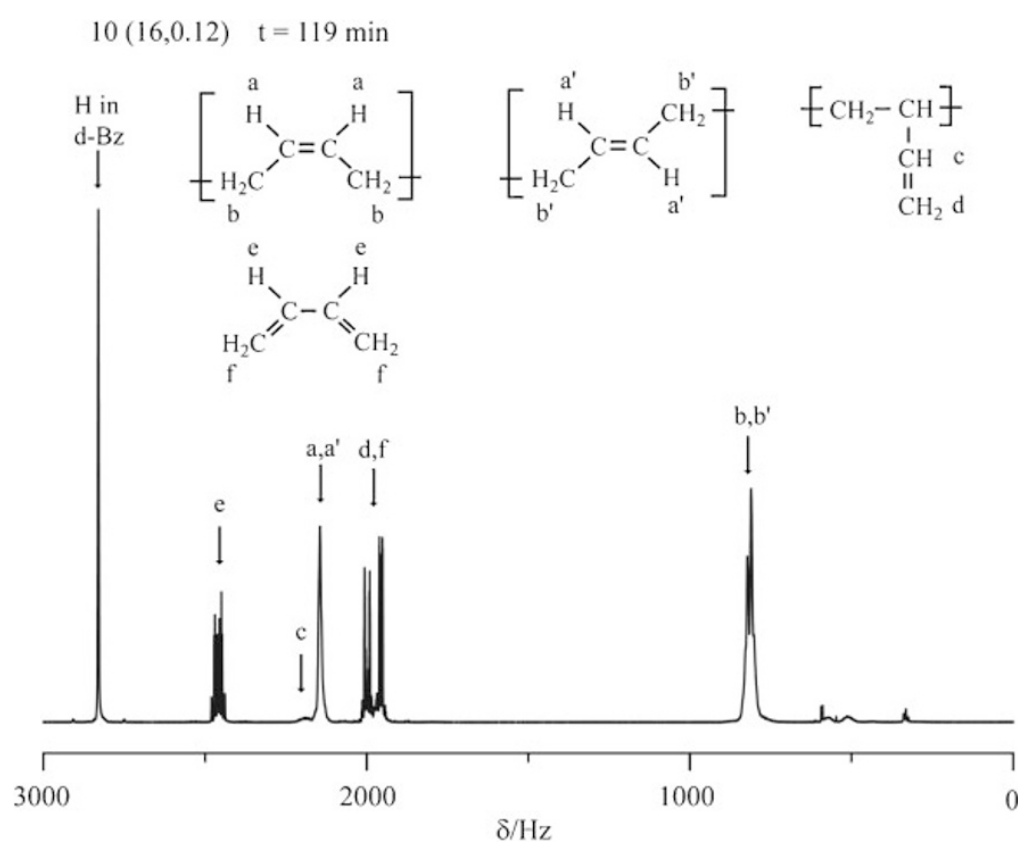

Figure 2. ${ }^{1} \mathrm{H}$ NMR spectrum of $10(16,0.12)$ polymerizing solution at $t=119 \mathrm{~min}$.

dPB concentration in the NMR tube $T_{3}$.

With the above procedure, we successfully prepared the $\mathrm{oBLi} / \mathrm{dPB} / \mathrm{Bz}$ solutions of nearly the same oBLi concentration but different dPB concentrations (including the zero concentration in $\mathrm{T}_{1}$ ) sealed in separate NMR tubes. The $\mathrm{hB}$ monomer (in the ampoule A) was distilled into the NMR tube chilled with liquid nitrogen (liq- $\mathrm{N}_{2}$ ) and then the tube was sealed off. The oBLi/dPB/Bz solution and $\mathrm{hB}$ monomer thus sealed in the tube was kept frozen with liq- $\mathrm{N}_{2}$ until they were subjected to the NMR measurement. Just before the measurement, they were allowed to quickly melt and mix with each other at room temperature and then the tube containing this mixture was quickly set in the NMR spectrometer to start the polymerization experiment at $30^{\circ} \mathrm{C}$.

\section{Measurements}

NMR For the $\mathrm{hB} / \mathrm{oBLi} / \mathrm{dBz}$ and $\mathrm{hB} / \mathrm{oBLi} / \mathrm{dPB} /$ $\mathrm{dBz}$ solutions kept at $30^{\circ} \mathrm{C}$ in the NMR tubes, the polymerization (propagation) process of the $\mathrm{hB}$ monomers was monitored with a JEOL JNM-AL400 spectrometer. The ${ }^{7} \mathrm{Li}$ and ${ }^{1} \mathrm{H}$ NMR measurements were conducted alternatively with respective data accumulation periods of $17 \mathrm{~min}$ and $4 \mathrm{~min}$ until the fraction of the residual monomer $\phi(t)$ decreased below 0.01 (i.e., conversion $>0.99$ ). The measured ${ }^{7} \mathrm{Li}$ and ${ }^{1} \mathrm{H}$ chemical shifts were expressed as the values relative to reference systems, the proton in the solvent $(0.5 \%$ in $\mathrm{dBz}$; internal reference) and $0.2 \mathrm{M} \mathrm{LiCl} / \mathrm{D}_{2} \mathrm{O}$ solution, respectively. (No ${ }^{1} \mathrm{H}$-decoupling was applied to the ${ }^{7} \mathrm{Li}$ spectra.) For this purpose, the spectrometer was alternatively operated under a static magnetic field of $9.4 \mathrm{~T}$ for respective measurements, and the resonance frequencies were $400.0 \mathrm{MHz}$ (for ${ }^{1} \mathrm{H}$ NMR) and $153.86 \mathrm{MHz}$ (for ${ }^{7} \mathrm{Li} \mathrm{NMR}$ ).

Those data were recorded as a function of the time $t$ from the onset of reaction (when the $\mathrm{hB} / \mathrm{oBLi} / \mathrm{dBz}$ and $\mathrm{hB} / \mathrm{oBLi} / \mathrm{dPB} / \mathrm{dBz}$ solutions were allowed to quickly melt/mix) to the midpoint of respective data accumulation periods. The ${ }^{7} \mathrm{Li}$ and ${ }^{1} \mathrm{H}$ spectra detected the chemical state of $\mathrm{Li}$ and the concentration of various ${ }^{1} \mathrm{H}$ species, respectively. An example of the ${ }^{1} \mathrm{H}$ spectra, obtained during the polymerizing process of hPB in the presence of dPB (a batch 10(16, 0.12) explained later), is shown in Figure 2. The ${ }^{1} \mathrm{H}$ signals from the $\mathrm{CH}_{2}$ and $\mathrm{CH}$ groups of the $\mathrm{hB}$ monomer and the polymerized $\mathrm{hPB}$ are well resolved at different chemical shifts. From the integrated intensities $I$ of those signals at respective times $t$, we evaluated the fraction of the residual $\mathrm{hB}$ monomer $\phi(t)=I_{\text {monomer }} /$ $\left(I_{\text {monomer }}+I_{\mathrm{hPB}}\right)$, as similar to the method utilized by Niu et al. ${ }^{10}$ The $\mathrm{dPB}$ and the oligomeric fragment of initiator (the oB part from oBLi), both made of $\mathrm{dB}$ monomers having the deuterated fraction $=0.99$, had a small contribution to these ${ }^{1} \mathrm{H}$ signals, and a minor correction for this contribution was made in the evaluation of $\phi(t)$. The use of $\mathrm{dPB}$ (not hPB) as the chemically inert matrix chains, combined with this correction, enabled us to follow the decay of $\phi(t)$ down to 0.01 (or even below) with a sufficient accuracy even in the presence of the matrix chains.

GPC After completion of the polymerization in the NMR tube, the tube was opened in an atmosphere of methanol vapor to terminate the living $\mathrm{hPBLi}$ chains therein. Then, the $\mathrm{dBz}$ solution of hPB (hPB 
plus dPB for some batches) was recovered in a clean glass jar, and the total PB concentration (in mass/volume unit) was determined from the masses before and after thorough evaporation of $\mathrm{dBz}$ in vacuum.

The hPB samples thus recovered from the polymerizing $\mathrm{hB} / \mathrm{oBLi} / \mathrm{Bz}$ solutions containing no $\mathrm{dPB}$ were dissolved in tetrahydrofuran (THF), and their molecular characteristics were determined from GPC (CO8020 and DP-8020, Tosoh). The eluent was THF, and monodisperse linear PB standards ${ }^{19}$ were utilized to calibrate the elution volume.

The GPC measurements were separately conducted also for the oligomeric initiator terminated with methanol as well as for the dPB samples, the latter utilized as the non-reactive additive in the polymerizing solutions. The GPC profiles of the dPB samples were utilized in the characterization of the hPB sample recovered from the polymerizing solutions containing $\mathrm{dPB}$, as explained below.

Figure 3 shows an example of the GPC profile (thin solid curve) for the mixtures of hPB and dPB recovered from such a solution containing dPB-1 $\left(M_{\mathrm{w}}=\right.$ $\left.15.5 \times 10^{3}\right)$. In the preparation of the polymerizing solutions, the molar ratio of the $\mathrm{hB}$ monomer and initiator was adjusted in a way that the fully polymerized hPB had a molecular weight considerably different from that of dPB. For this reason, the GPC profiles of all hPB/dPB mixtures examined in this study had two peaks and the portion of the profile around the dPB peak agreed well with the known profile for $\mathrm{dPB}$; see the dotted curve in Figure 3. Thus, the GPC profiles of the hPB samples (thick dashed curve) were easily evaluated by subtracting the dPB profile from the raw GPC profile (thin solid curve) and the hPB samples were characterized with this subtracted profiles. In addition, the mass ratio of the $\mathrm{hPB}$ and
dPB samples was evaluated from the ratio of the GPC peak areas for respective samples.

Table I summarizes the characteristics of the hPB samples and their polymerization condition. The first number in the code indicates the hPB molecular weight (in unit of 1000) on completion of the polymerization, and the numbers in the parenthesis represent the molecular weight and concentration of $\mathrm{dPB}$ added to the hPB polymerization system. The number " 0 " in the parenthesis means that no dPB was added to the system.

The molecular weights determined for the hPB samples agreed well with those expected from the known molar ratio of the $\mathrm{hB}$ monomer and oBLi (initiator). This result demonstrated that our polymerizing solutions contained no detectable impurities (because of the rinsing operation with the DPELi solution and the repeated precipitation/dissolution procedure for the dPB samples explained earlier).

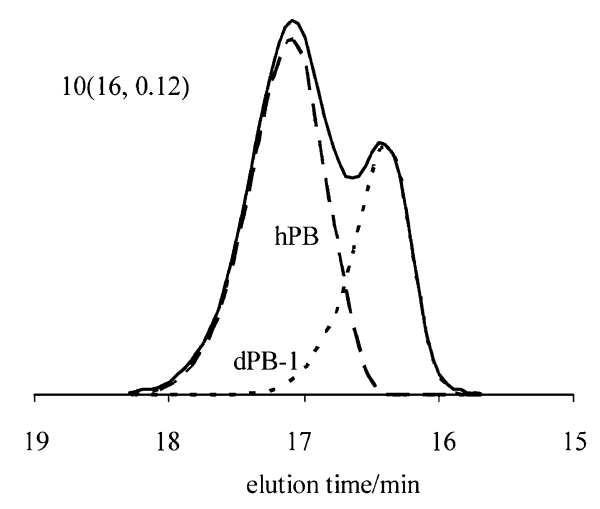

Figure 3. GPC chromatogram of 10(16,0.12) solution on completion of polymerization (thin solid curve). Dashed and dotted curves indicate the chromatograms for the polymerized hPB and matrix $\mathrm{dPB}$, respectively.

Table I. Characteristics of hPB samples polymerized at $30^{\circ} \mathrm{C}$

\begin{tabular}{|c|c|c|c|c|c|}
\hline Batch Code $^{\mathrm{a}}$ & $10^{-3} M_{\mathrm{w}}$ & $M_{\mathrm{w}} / M_{\mathrm{n}}$ & $C(\infty)^{\mathrm{b}} / \mathrm{g} \mathrm{cm}^{-3}$ & $C_{\mathrm{m}}{ }^{\mathrm{c}} / \mathrm{g} \mathrm{cm}^{-3}$ & $10^{-3} M_{\mathrm{w}, \mathrm{dPB}}$ \\
\hline \multicolumn{6}{|c|}{ polymerized in the absence of $\mathrm{dPB}$} \\
\hline $9(0)$ & 8.6 & 1.04 & 0.171 & 0 & \\
\hline 11(0) & 11.2 & 1.04 & 0.160 & 0 & \\
\hline $17(0)$ & 17.3 & 1.04 & 0.153 & 0 & \\
\hline $25(0)$ & 25.1 & 1.04 & 0.105 & 0 & \\
\hline \multicolumn{6}{|c|}{ polymerized in the presence of $\mathrm{dPB}$} \\
\hline $10(16,0.12)$ & 9.6 & 1.05 & 0.189 & 0.115 & 15.5 \\
\hline $12(49,0.04)$ & 11.9 & 1.04 & 0.170 & 0.039 & 48.7 \\
\hline $22(60,0.05)$ & 22.4 & 1.05 & 0.120 & 0.045 & 60.0 \\
\hline $26(60,0.10)$ & 26.3 & 1.07 & 0.100 & 0.095 & 60.0 \\
\hline
\end{tabular}

${ }^{a}$ The first number in the code indicates the hPB molecular weight (in unit of 1000) on completion of the polymerization, and the numbers in the parenthesis represent the molecular weight and concentration of $\mathrm{dPB}$ added to the hPB polymerization system. The number " 0 " in the parenthesis means that no dPB was added to the system. ${ }^{\mathrm{b}}$ Concentration of $\mathrm{hPB}$ on completion of polymerization. ${ }^{\mathrm{c}}$ Concentration of dPB during the polymerization of $\mathrm{hPB}$. 

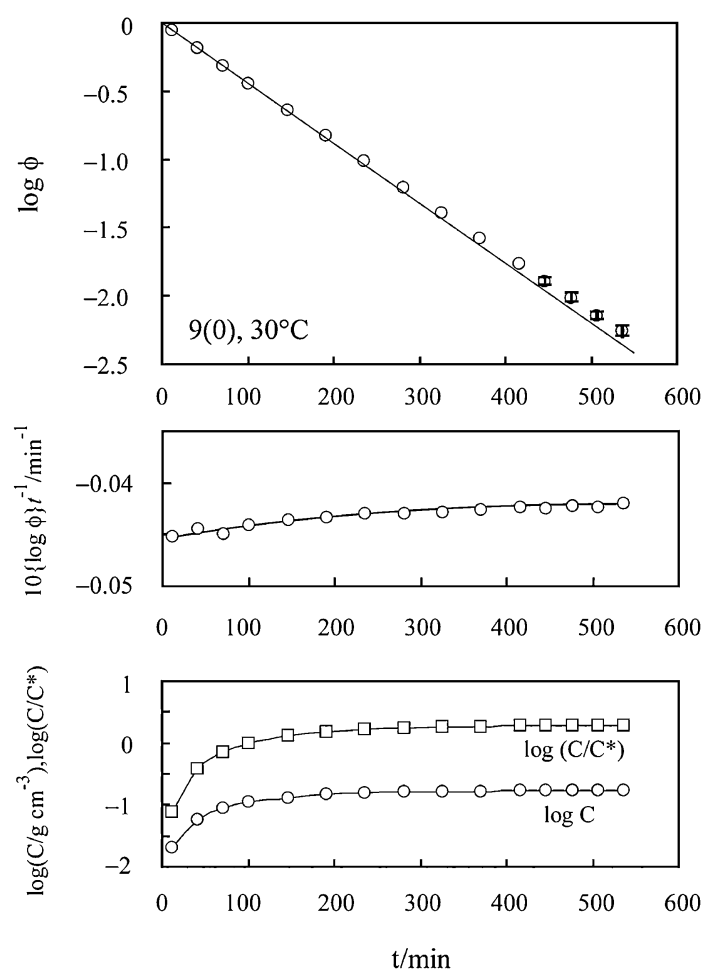

Figure 4. Propagation behavior of $9(0)$ solution at $30^{\circ} \mathrm{C}$. Top panel; semi-logarithmic plot of residual monomer fraction $\phi(t)$ against time $t$, middle panel; change of $10\{\log \phi\} t^{-1}$ with $t$, bottom panel; changes of concentration $C$ (circles) and $C / C^{*}$ ratio (squares) of polymerizing hPB with $t$.

\section{RESULTS AND DISCUSSION}

\section{Overview of NMR Data of Solutions without $d P B$}

For the 9(0), 11(0), 17(0), and 25(0) solutions containing no dPB ( $c f$. Table I), the residual monomer faction $\phi(t)$ is semi-logarithmically plotted against the polymerization time $t$ in the top panels of Figures 4-7, respectively. For the data points in these panels indicated without error bars, the uncertainties in the $\log \phi(t)$ values were smaller than the size of the symbols. Since the oligomeric oBLi having the butadienyllithium end was utilized as the initiator, the decay of $\phi(t)$ is exclusively attributed to the propagation process (no contribution from the initiating reaction of $s$ - $\mathrm{BuLi}$ with $\mathrm{B}$ monomer). In this point, our experiment is different from that by Niu et al. ${ }^{10}$ (who utilized slowly initiating $t$-butyllithium in heptane to follow $\phi(t)$ in the initiation and propagation processes.)

For the conventional molecular picture shown in Scheme 1, the propagation occurs only through the dissociated chains (being in equilibrium with the aggregates) and $\phi(t)$ is expected to exhibit a universal single-exponential decay when plotted against a normalized time $t_{\mathrm{n}}(c f$. eq 3$)$ :
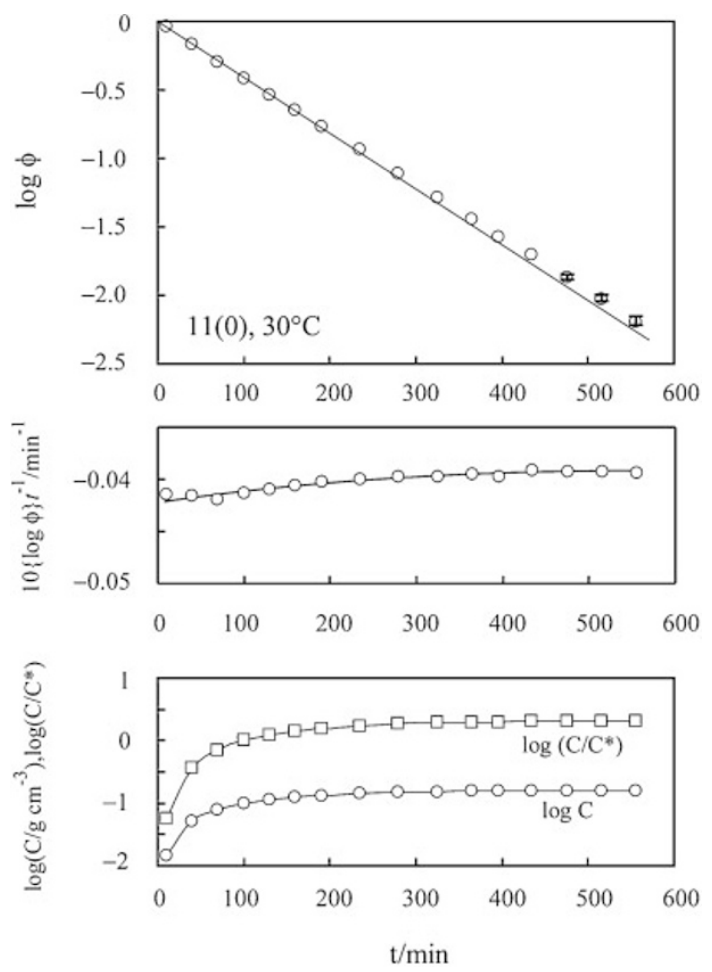

Figure 5. Propagation behavior of $11(0)$ solution at $30^{\circ} \mathrm{C}$. Top panel; semi-logarithmic plot of residual monomer fraction $\phi(t)$ against time $t$, middle panel; change of $10\{\log \phi\} t^{-1}$ with $t$, bottom panel; changes of concentration $C$ (circles) and $C / C^{*}$ ratio (squares) of polymerizing hPB with $t$.
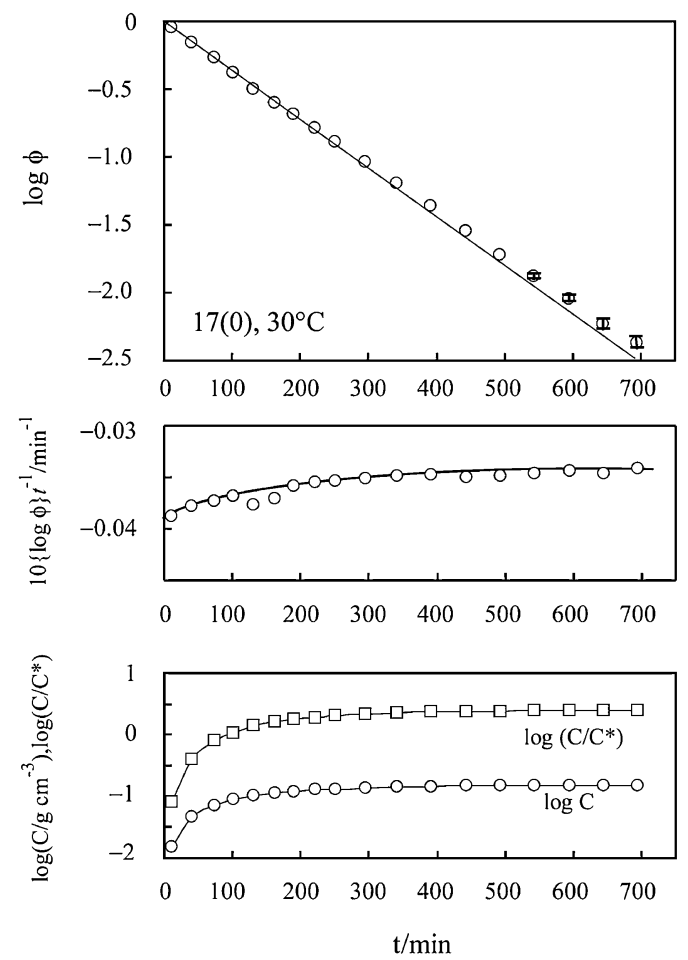

Figure 6. Propagation behavior of $17(0)$ solution at $30^{\circ} \mathrm{C}$. Top panel; semi-logarithmic plot of residual monomer fraction $\phi(t)$ against time $t$, middle panel; change of $10\{\log \phi\} t^{-1}$ with $t$, bottom panel; changes of concentration $C$ (circles) and $C / C^{*}$ ratio (squares) of polymerizing hPB with $t$. 

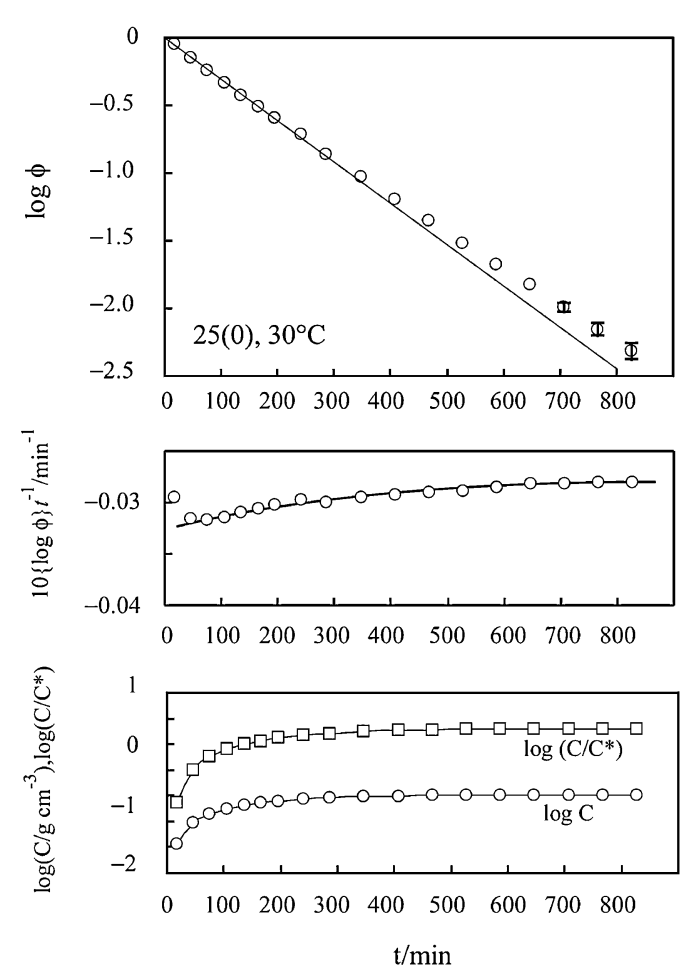

Figure 7. Propagation behavior of $25(0)$ solution at $30^{\circ} \mathrm{C}$. Top panel; semi-logarithmic plot of residual monomer fraction $\phi(t)$ against time $t$, middle panel; change of $10\{\log \phi\} t^{-1}$ with $t$, bottom panel; changes of concentration $C$ (circles) and $C / C^{*}$ ratio (squares) of polymerizing hPB with $t$.

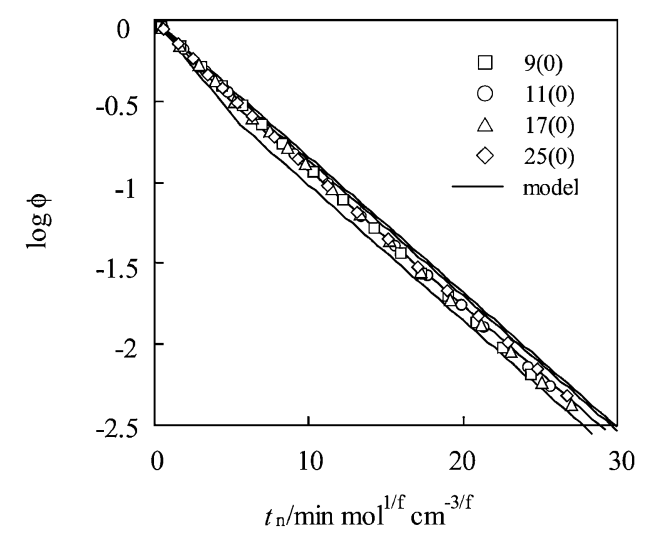

Figure 8. Semi-logarithmic plots of residual monomer fraction $\phi(t)$ in polymerizing solutions containing no dPB against normalized time, $t_{\mathrm{n}}=t\left\{[\mathrm{I}]_{0} / f\right\}^{1 / f}$ with $f=4$. Solid curves indicate $\phi(t)$ calculated from a model described in the text. Note that the measured as well as calculated $\phi(t)$ are almost universally dependent on $t_{\mathrm{n}}$.

$$
\phi(t)=\exp \left(-k_{\mathrm{p}} K_{\mathrm{d}}^{1 / f} t_{\mathrm{n}}\right) \quad \text { with } \quad t_{\mathrm{n}}=\left\{[\mathrm{I}]_{0} / f\right\}^{1 / f} t
$$

In Figure 8, the $\log \phi(t)$ data shown in Figures 4-7 are re-plotted against this $t_{\mathrm{n}}$, with the initiator (oBLi) concentration $[\mathrm{I}]_{0}$ included in $t_{\mathrm{n}}$ being determined from the hPB concentration and molecular weights on completion of the polymerization. For the average aggregation number estimated previously, ${ }^{15,16} f=4$, the data points are mostly collapsed onto a universal curve within small experimental scatters. Furthermore, in the top panels of Figures 4-7, the data points in a range of $\phi(t) \geq 0.1$ are well described by the solid lines representing the single-exponential decay for these points, which is in harmony with the previous studies for polydienes ${ }^{1-8}$ that mostly examined the propagation kinetics in this range of $\phi(t)$.

However, in the top panels of Figures 4-7, we also note that the decay of $\log \phi(t)$ data becomes a little slower at long $t$ where $\phi(t)<0.1$. (The decay in the entire range of $t$ appears to be described by a sum of two or more exponential decay terms.) This small but non-negligible deviation from the single-exponential decay is more clearly noted for the $\{\log \phi(t)\} / t$ ratio shown in the middle panels: This ratio should be constant for the single-exponential decay but our polymerizing solutions exhibit a moderate but nonnegligible increase of the ratio.

For investigation of the molecular origin of this non-conventional behavior, we estimated a degree of overlapping of the star-like $f$-mer aggregates (the main component in the solutions) in the following way. First, we utilized the $\phi(t)$ data to evaluate the concentration $C(t)$ and molecular weight $M(t)$ of the hPB chains in the solutions at time $t$ during the polymerization (propagation) process as

$$
C(t)=C(\infty)\{1-\phi(t)\}, \quad M(t)=M(\infty)\{1-\phi(t)\}
$$

Here, $C(\infty)$ and $M(\infty)$ indicates the hPB concentration and molecular weight on completion of the polymerization ( $c f$. Table I). From an empirical equation reported in literature ${ }^{20}$ and the $M(t)$ value obtained as above, the root-mean-square radius of gyration of the hPB chain in the dissociated form was evaluated as

$$
R_{\mathrm{d}}(t)=3.79 \times 10^{-2} M(t)^{1 / 2} \quad(\text { in } \mathrm{nm})
$$

Here, we have neglected the excluded volume effect in benzene for our hPB chains with relatively small $M$. (In fact, the $R_{\mathrm{d}}$ value of those chains evaluated from the empirical equation in the good solvent limit ${ }^{20}$ was not significantly different from that given by eq 6.) The $R_{\mathrm{d}}(t)$ and $M(t)$ values allowed us to estimate the overlapping concentration for the star-like $f$-mer aggregates (main component in the solution): ${ }^{16,21}$

$$
C^{*}(t)=F \frac{\left\{M(t) / N_{\mathrm{A}}\right\}}{\left\{4 \pi R_{\mathrm{d}}^{3}(t) / 3\right\}} \quad \text { with } \quad F=\frac{f^{5 / 2}}{(3 f-2)^{3 / 2}}
$$

In the bottom panels of Figures 4-7, squares show the $C(t) / C^{*}(t)$ ratio thus evaluated for $f$-mer aggregates with $f=4$. As the polymerization proceeds, both of $C(t)$ (circles) and $M(t)$ (not shown) increases 


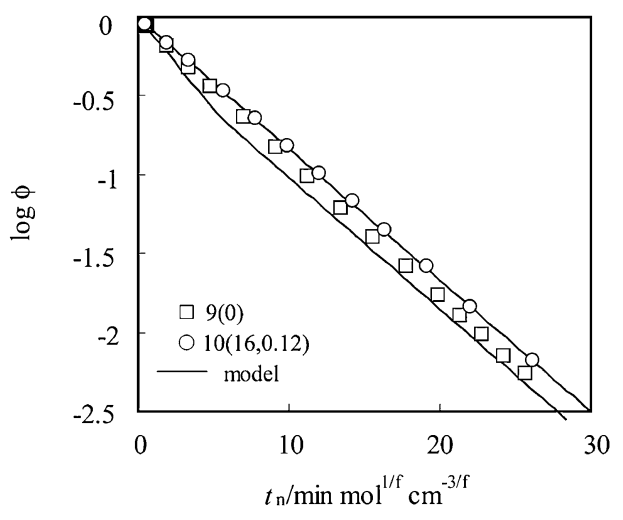

Figure 9. Comparison of decay of residual monomer fraction $\phi(t)$ for the $9(0)$ and $10(16,0.12)$ solutions having nearly the same monomer/initiator compositions but different dPB concentrations. Solid curves indicate $\phi(t)$ calculated from a model described in the text.

to give a significant increase of $C(t) / C^{*}(t)\left(\sim C M^{1 / 2}\right.$; $c f$. eq 7). Comparing the top and bottom panels of Figures 4-7, we note that the conventional, singleexponential decay of $\phi(t)$ at short $t$ (where $\phi>0.1$ ) begins to fail to describe the $\phi(t)$ data as the $C(t) /$ $C^{*}(t)$ ratio becomes considerably larger than unity, i.e., when the $f$-mer aggregates begin to overlap considerably. This result suggests that the osmotic interaction of these aggregates affects the decay of $\phi(t)$. In the next section, this effect is further examined for the polymerizing solution containing $\mathrm{dPB}$ chains.

\section{NMR Data for Solutions Containing $d P B$}

Figure 9 compares the residual hB monomer fraction $\phi(t)$ determined for the 10(16,0.12) and 9(0) solutions, with the former containing the dPB-1 matrix chains $\left(M=15.5 \times 10^{3}\right)$ at the concentration of $C_{\mathrm{m}}=0.115 \mathrm{~g} \mathrm{~cm}^{-3}$ while the latter containing no matrix. The matrix chains are chemically inert for the polymerization. The two solutions had nearly the same concentrations of the initiator (oBLi) and $\mathrm{hB}$ monomer at $t=0$ and thus gave hPB of nearly the same $C(\infty)$ and $M(\infty)$ on completion of the polymerization; see Table I. Figures 10 and 11 compare $\phi(t)$ for the other sets of the solutions with/without matrix dPB giving nearly the same $C(\infty)$ and $M(\infty)$. For all solutions containing $\mathrm{dPB}, C_{\mathrm{m}}$ is larger than the overlapping concentration $C_{\mathrm{m}}{ }^{*}$ of respective dPB chains. Thus, in these solutions, the polymerization of hPB occurred in an osmotic mesh formed by the $\mathrm{dPB}$ chains (as well as by the hPB chains themselves).

For all sets of the solutions with/without dPB, we note that the decay of $\phi(t)$ is moderately retarded in the presence of the matrix dPB chains, and this retardation is more prominent for the solutions polymerizing low $M \mathrm{hPB}$ (Figures 9 and 10) than that giving high $M$ hPB (Figure 11).

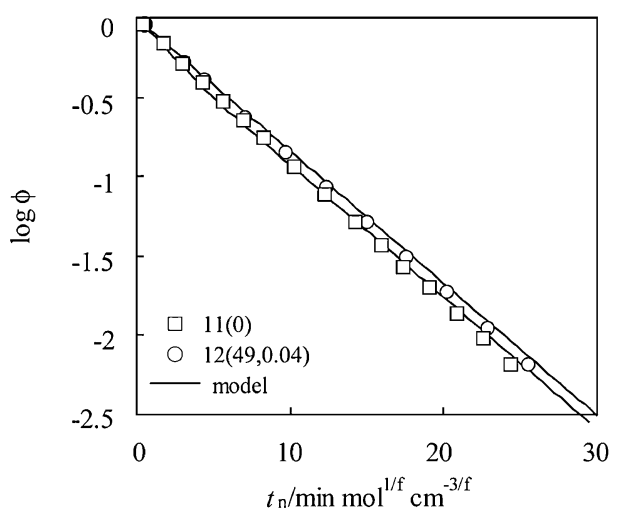

Figure 10. Comparison of decay of residual monomer fraction $\phi(t)$ for the 11(0) and 12(49,0.04) solutions having nearly the same monomer/initiator compositions but different dPB concentrations. Solid curves indicate $\phi(t)$ calculated from a model described in the text.

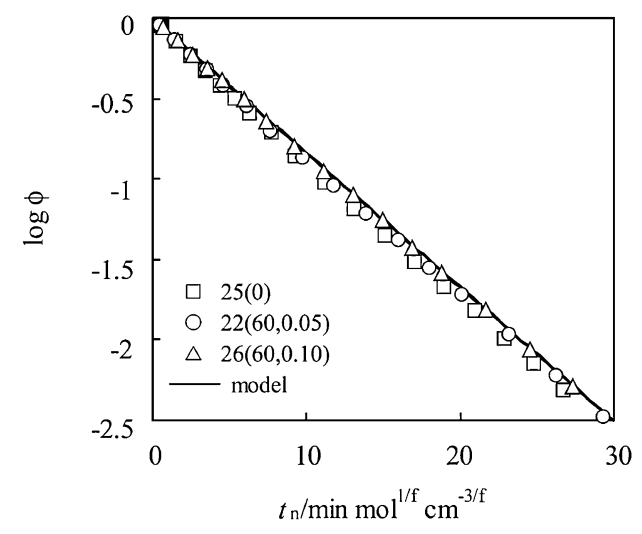

Figure 11. Comparison of decay of residual monomer fraction $\phi(t)$ for the 25(0), 22(60,0.05) and 26(60,0.10) solutions having nearly the same monomer/initiator compositions but different $\mathrm{dPB}$ concentrations. Solid curves indicate $\phi(t)$ calculated from a model described in the text.

The observed retardation can be related, in principle, to several effects of the dPB chains on the anionic hPBLi chains, (1) a change in the chemical environment (polarity) affecting the reactivity of the dissociated hPBLi chains, (2) a decrease of the mobility of the dissociated hPBLi chains, (3) a shift of the equilibrium between the dissociated hPBLi chains and the $(\mathrm{hPBLi})_{f}$ aggregates, and (4) an enhancement of the osmotic field for hPBLi. However, the effect (1) does not seem to be important because $\mathrm{dPB}$ is almost equally nonpolar compared to the solvent, dBz. The effect (2) should be also irresponsible for the observed retardation because the $\mathrm{hB}$ monomers are highly mobile and thus the propagation reaction would require no large-scale motion of the dissociated chains. (Note that the molar concentration is much higher for the monomers than for the hPBLi chains even at the longest $t$ covered in our experiments and the propagation for the dissociated chains should be dominated by the 
motion of the monomers.)

As for the above effect (3), we note that the equilibrium between the dissociated $\mathrm{hPBLi}$ chains and the $(\mathrm{hPBLi})_{f}$ aggregates could be, in principle, shifted toward the aggregates on an increase of the total polymer concentration due to the presence of the matrix $\mathrm{dPB}$ chains. If this shift (resulting in the decrease of the concentration of the dissociated hPBLi chains) is mainly responsible for the retarded decay of $\phi(t)$ in the presence of $\mathrm{dPB}$, the magnitude of this retardation should be larger at a higher matrix concentration $C_{\mathrm{m}}$ and similar for the solutions having similar $C_{\mathrm{m}}$ and $C(\infty)$ values irrespective of the molecular weight of the polymerizing hPB. However, this is not the case: The magnitude of the retardation seen in Figure 11 is just comparable for the increases of $C_{\mathrm{m}}$ from 0 to $0.045 \mathrm{~g} \mathrm{~cm}^{-3}$ (from square to circle) and from 0.045 to $0.095 \mathrm{~g} \mathrm{~cm}^{-3}$ (from circle to triangle), and the retardation is more significant for the solution polymerizing low- $M$ hPB (Figures 9 and 10) than that giving high- $M$ hPB (Figure 11). Thus, the above shift of the equilibrium may have occurred to some extent but cannot be the main factor giving the dPB-induced retardation of the $\phi(t)$ decay.

On the basis of these considerations, we hereafter focus on the effect (4), the enhancement of the osmotic environment due to the matrix dPB chains. The observed retardation due to $\mathrm{dPB}$ cannot be mainly attributed to the osmotic effect on the concentration of the dissociated chains (shift of the equilibrium between $\mathrm{hPBLi}$ and $(\mathrm{hPBLi})_{f}$ ), as discussed above. In other words, this effect is to be considered for a propagating species other than the dissociated chains. Thus, as the propagating species sensitive to the osmotic effect, we first consider the fused $2 f$-mer aggregate (Scheme 2) that appears to play an important role in the Li-Li exchange process. ${ }^{16}$ The $2 f$-mer aggregate is less stable/ more reactive than the $f$-mer aggregates (as suggested from the previous ${ }^{7} \mathrm{Li}$ NMR study ${ }^{16}$ ) and is the simplest candidate of this propagating species. For completeness, we will also discuss the contribution of the other species (such as $n f$-mer and $f / 2$-mer aggregates) to the polymerization.

The osmotic effect was indeed confirmed qualitatively for our polymerizing solutions from their ${ }^{7}$ Li NMR spectra. All solutions had similar spectra, and representative spectra obtained for the 10(16, 0.12 ) and 9(0) solutions are shown in Figure 12. These spectra were obtained at representative times during the polymerization process, and their intensities are normalized. At our experimental temperature, $30^{\circ} \mathrm{C}$, the exchange of the $\mathrm{Li}$ species in differently chemical states is not sufficiently slow compared to the NMR time scale. For this reason, the spectra do not exhibit multiple peaks that correspond to respec-

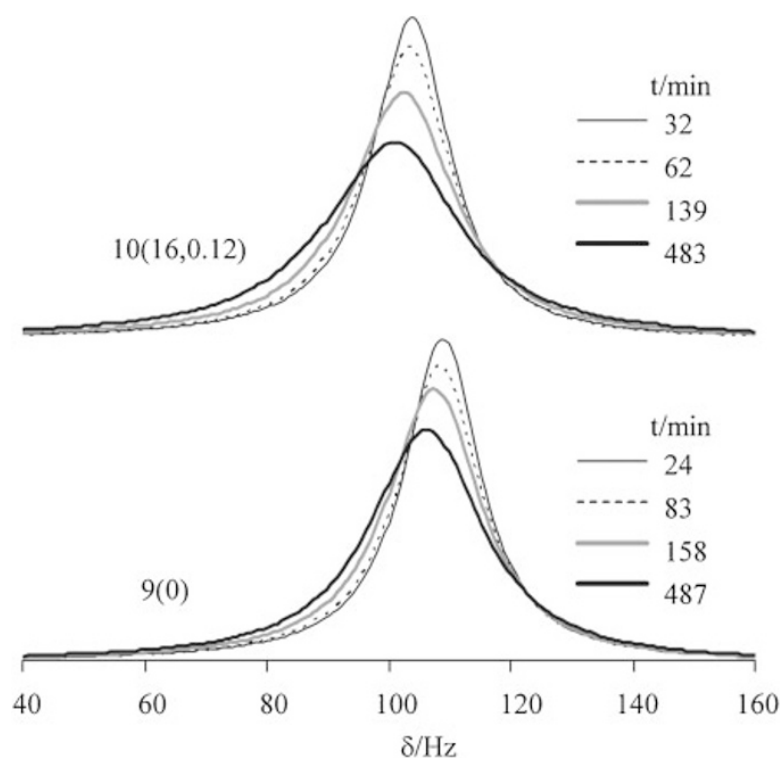

Figure 12. ${ }^{7} \mathrm{Li}$ NMR spectra of $9(0)$ and $10(16,0.12)$ polymerizing solutions having nearly the same monomer/initiator compositions but different dPB concentrations. The spectra are shown at representative times during the propagation process.

tive Li species (seen previously at lower temperatures $^{16}$ ) and the Li-Li exchange time cannot be evaluated quantitatively from the spectra. However, even for the unimodal spectra seen in Figure 12, we note a characteristic feature that the spectrum is broader for the 10(16,0.12) solution containing dPB than for the 9(0) solution without dPB. This difference is most clearly noted at long times, 483 and $487 \mathrm{~min}$ : The width at the half-maximum at these times is 20.3 and $17.1 \mathrm{~Hz}$ for the $10(16,0.12)$ and $9(0)$ solutions, respectively. In addition, the spectrum of each solution becomes broader with increasing $t$. These features are attributable to retardation of the $\mathrm{Li}-\mathrm{Li}$ exchange due to an enhancement of the osmotic penalty for the fusion of the aggregates, as discussed previously. ${ }^{16}$ In our solutions, the penalty is enhanced in the presence of $\mathrm{dPB}$ and on the progress of polymerization (on the increase of $C(t)$ and $M(t)$ of the hPB chain).

This ${ }^{7} \mathrm{Li}$ NMR observation lends qualitative support to our simple molecular picture that the fused $2 f$-mer aggregates behave as the osmotically affected propagating species. Thus, in the next section, we formulate a simple model for competitive propagation through the fused $2 f$-mer aggregates and dissociated chains and compare the behavior of this model with the experiments. After this comparison, we will also discuss the contribution of the other species (such as $n f$-mer and $f / 2$-mer aggregates) to the propagation.

\section{Model for Unimer/f-mer/2f-mer Systems}

Kinetics We consider the simplest kinetics for three species, the dissociated chain (unimer), $f$-mer 
aggregates, and the fused $2 f$-mer aggregates; $c f$. Scheme 2: Both of the dissociated chains and $2 f$ mer aggregates are in equilibrium with the $f$-mer aggregates. The $f$-mer aggregates are well stabilized and assumed to be chemically inert. The $2 f$-mer aggregates are more reactive and considered to be a propagating species that competes with the dissociated chains. The osmotic effect emerges for the propagation through the $2 f$-mer aggregate, because the fusion into this aggregate unavoidably increases a local concentration thereby enhancing the osmotic free energy.

For this kinetic picture, the monomer consumption rate can be described by

$$
\frac{\mathrm{d}[\mathrm{M}]}{\mathrm{d} t}=-k_{\mathrm{p}}[\mathrm{P}][\mathrm{M}]-k_{\mathrm{p}}{ }^{\prime}\left[\mathrm{P}_{2 f}\right][\mathrm{M}]
$$

Here, $k_{\mathrm{p}}$ and $k_{\mathrm{p}}{ }^{\prime}$ are the propagation rate constants for the dissociated chain and $2 f$-mer aggregate, respectively, and $[\mathrm{P}]$ and $\left[\mathrm{P}_{2 f}\right]$ are the molar concentrations of these species. Equation 8 reduces to the conventional eq 1 in the absence of the fusion-aided propagation $\left(k_{\mathrm{p}}{ }^{\prime}=0\right)$. Considering that the equilibrium is predominantly in the $f$-mer aggregate state, we can express $[\mathrm{P}]$ and $\left[\mathrm{P}_{2 f}\right]$ in terms of respective equilibrium constants $\left(K_{\mathrm{d}}\right.$ and $K_{\mathrm{d}}{ }^{\prime} ; c f$. Scheme 2$)$, the initiator (oBLi) concentration $[\mathrm{I}]_{0}$ just before the propagation, and the aggregation number $f(=4)$ as

$$
\begin{aligned}
{[\mathrm{P}] } & =\left\{K_{\mathrm{d}}\left[\mathrm{P}_{f}\right]\right\}^{1 / f} \cong\left\{\frac{K_{\mathrm{d}}[\mathrm{I}]_{0}}{f}\right\}^{1 / f}, \\
{\left[\mathrm{P}_{2 f}\right] } & =\frac{\left[\mathrm{P}_{f}\right]^{2}}{K_{\mathrm{d}^{\prime}}} \cong \frac{[\mathrm{I}]_{0}^{2}}{K_{\mathrm{d}} f^{2}}
\end{aligned}
$$

From eqs 8 and 9 , a time evolution equation for the residual monomer fraction $\phi(t)\left(=[\mathrm{M}] /[\mathrm{M}]_{0}\right)$ is obtained as

$$
\frac{\mathrm{d}\{\ln \phi(t)\}}{\mathrm{d} t}=-k_{\mathrm{p}} K_{\mathrm{d}}^{1 / f}\left\{\frac{[\mathrm{I}]_{0}}{f}\right\}^{1 / f}-\left\{\frac{k_{\mathrm{p}}{ }^{\prime}}{K_{\mathrm{d}^{\prime}}}\right\}\left\{\frac{[\mathrm{I}]_{0}}{f}\right\}^{2}
$$

The equilibrium constant between the $2 f$-mer and $f$ mer aggregates can be expressed as a product of a non-osmotic contribution $K_{\mathrm{d} 0}{ }^{\prime}$ and an osmotic contribution, $\exp \left(\Delta G_{\mathrm{os}}(t) / k_{\mathrm{B}} T\right)$. Here, $\Delta G_{\mathrm{os}}(t)$ is the osmotic free energy increment on fusion of two $f$-mer aggregates into a $2 f$-mer aggregate at a time $t, k_{\mathrm{B}}$ is the Boltzmann constant, and $T$ is the absolute temperature. Thus, eq 10 is rewritten as

$$
\frac{\mathrm{d}\{\ln \phi(t)\}}{\mathrm{d} t}=-\frac{1}{\tau}-B \exp \left(-\frac{\Delta G_{\mathrm{os}}(t)}{k_{\mathrm{B}} T}\right)
$$

with

$$
\tau=\frac{1}{k_{\mathrm{p}} K_{\mathrm{d}}^{1 / f}}\left\{\frac{f}{[\mathrm{I}]_{0}}\right\}^{1 / f} \text { and } B=\left\{\frac{k_{\mathrm{p}}{ }^{\prime}}{K_{\mathrm{d} 0^{\prime}}}\right\}\left\{\frac{[\mathrm{I}]_{0}}{f}\right\}^{2}
$$

Note that the rate constants $k_{\mathrm{p}}$ and $k_{\mathrm{p}}{ }^{\prime}$ and the non-

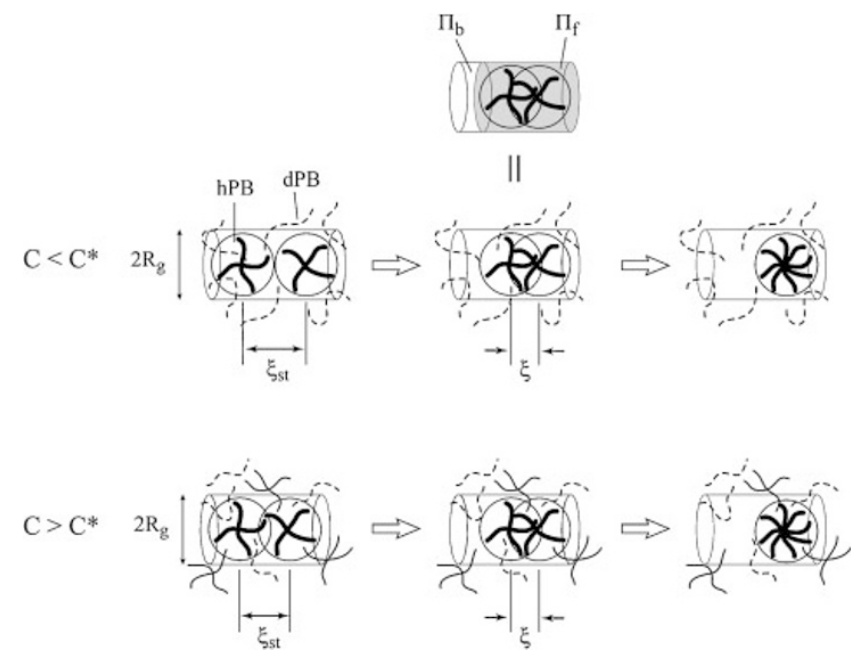

Figure 13. Schematic illustration of a geometry considered for calculation of the osmotic free energy increment on fusion of $f$-mer aggregates.

osmotic equilibrium constants $K_{\mathrm{d}}$ and $K_{\mathrm{d} 0}{ }^{\prime}$ are functions of $T$ but independent of the concentrations and molecular weights of the propagating hPBLi chains and matrix $\mathrm{dPB}$ chains.

Osmotic Factor For evaluation of $\Delta G_{\text {os }}$, we consider a geometry illustrated in Figure 13. The matrix dPB chains have a constant concentration $C_{\mathrm{m}}$ (larger than their $C_{\mathrm{m}}{ }^{*}$ ) and osmotically affect the fusion into the $2 f$-mer aggregate. $\Delta G_{\text {os }}$ increases when two $f$ mer aggregates approach each other (from their average separation) to enhance their mutual overlapping.

We may express this $\Delta G_{\text {os }}$ in terms of the osmotic pressures $\Pi_{\mathrm{f}}$ and $\Pi_{\mathrm{b}}$ in the forward and backward regimes shown in Figure 13, the separation $\xi$ between the centers of the two approaching $f$-mer aggregates, and the radius of gyration $R_{\mathrm{g}}$ of each aggregate as

$$
\Delta G_{\mathrm{os}}=-\pi R_{\mathrm{g}}^{2} \int_{\xi_{\mathrm{st}}}^{0}\left\{\Pi_{\mathrm{f}}-\Pi_{\mathrm{b}}\right\} \mathrm{d} \xi
$$

Here, the front factor $\pi R_{\mathrm{g}}{ }^{2}$ represents the cross-sectional area of those regimes, and $\xi_{\text {st }}$ indicates the center-to-center separation at the onset of the increase of the osmotic free energy: $\xi_{\text {st }}$ is chosen to be

$$
\begin{aligned}
& \text { for } C<C^{*}: \quad \xi_{\mathrm{st}}=2 R_{\mathrm{g}} \\
& \text { for } C \geq C^{*}: \quad \xi_{\text {st }}=2(3 / 4 \pi)^{1 / 3}\left\{f M / N_{\mathrm{A}} C\right\}^{1 / 3} \\
& =2 R_{\mathrm{g}}\left(C^{*} / C\right)^{1 / 3}
\end{aligned}
$$

These choices are based on the following argument: If the $f$-mer aggregates (main component in the system) have the concentration $C$ smaller than their own overlapping concentration $C^{*}$, these aggregates are dilute and the osmotic free energy begins to increase significantly only after the two aggregates approaches the touching location at $\xi_{\text {st }}=2 R_{\mathrm{g}}$ (eq 14a). ${ }^{16}$ On the other 
hand, for $C>C^{*}$, an approach of these aggregates by any magnitude enhances the overlapping to give this increase. For this case, $\xi_{\text {st }}$ is essentially identical to the equilibrium separation of the two aggregates, $\left\{f M / N_{\mathrm{A}} C\right\}^{1 / 3}$. The front factor of $2(3 / 4 \pi)^{1 / 3}(\cong 1.24)$ has been introduced in eq $14 \mathrm{~b}$ in order to ensure the continuity of $\xi_{\text {st }}$ at $C=C^{*}$.

Utilizing the standard semidilute formula, ${ }^{22,23}$ we may express the osmotic pressures $\Pi_{\mathrm{f}}$ and $\Pi_{\mathrm{b}}$ in the forward and backward regimes (Figure 13) in terms of the total polymer concentration $C_{\mathrm{f}}$ and $C_{\mathrm{b}}$ in respective regimes as

$$
\Pi_{\alpha}=K_{\Pi} \frac{C_{\alpha} R T}{f M}\left(\frac{C_{\alpha}}{C^{*}}\right)^{\frac{1}{3 v-1}} \text { with } \quad \alpha=\mathrm{f}, \mathrm{b}
$$

Here, $R$ and $T$ are the gas constant and absolute temperature, $v$ is the molecular weight exponent for $R_{\mathrm{g}}$ $(v \cong 1 / 2$ for our hPB having relatively small $M$ ), $K_{\Pi}$ is a constant close to unity, and the factor $f M$ is the molecular weight of the $f$-mer aggregate. This $f M$ factor appears in eq 15 because the molecular weight $f M$ included in $C^{*}$ should be cancelled to give the $M$-independent $\Pi_{\alpha}$. (We may utilize the overlapping concentration of the $\mathrm{dPB}$ chain in eq 15 instead $C^{*}$ of the aggregates. Then, eq 15 includes the molecular weight of this chain, instead of $f M$, but the results of analysis explained below are not affected by this change.)

The concentrations $C_{\mathrm{f}}$ and $C_{\mathrm{b}}$ in the forward and backward regimes are different from the overall concentration of the polymers (dPB plus hPB) in the whole solution, $C_{\mathrm{m}}+C$. The $\xi$ dependencies of $C_{\mathrm{f}}$ and $C_{\mathrm{b}}$ can be found from a geometrical consideration: For the case of $C<C^{*}$, the combined forward plus backward regime with $\xi<\xi_{\text {st }}$ contains just two $f$-mer aggregates (and the matrix $\mathrm{dPB}$ chains) and these aggregates are localized/squeezed in the forward regime as the fusion proceeds; see Figure 13. Thus, the backward regime contains no aggregate and the concentration in this regime is give by

$$
C_{\mathrm{b}}=C_{\mathrm{m}} \quad\left(\text { for } C<C^{*}\right)
$$

From a mass conservation relationship, $C_{\mathrm{f}}\left(2 R_{\mathrm{g}}+\xi\right)+$ $C_{\mathrm{b}}\left(\xi_{\mathrm{st}}-\xi\right)=\left(C_{\mathrm{m}}+C^{*}\right)\left(2 R_{\mathrm{g}}+\xi_{\mathrm{st}}\right) \quad$ with $\quad \xi_{\mathrm{st}}=2 R_{\mathrm{g}}$ (cf., eq 14a), we find an expression of $C_{\mathrm{f}}$,

$$
C_{\mathrm{f}}=C_{\mathrm{m}}+C^{*} \frac{4 R_{\mathrm{g}}}{\xi+2 R_{\mathrm{g}}} \quad\left(\text { for } C<C^{*}\right)
$$

We can similarly find the $\xi$ dependence of $C_{\mathrm{b}}$ for $C>C^{*}$ where the combined regime contains more than two $f$-mer aggregates. Considering that one of these aggregates are squeezed in the forward regime and the other aggregates are fixed in space ( $c f$. Figure 13) and further requiring the continuity of $C_{\mathrm{b}}$ (coincidence of $C_{\mathrm{b}}$ with that given by eq 16a) in the limit of $C \rightarrow C^{*}$, we find an expression of $C_{\mathrm{b}}$ for the backward regime:

$$
C_{\mathrm{b}}=C_{\mathrm{m}}+C-C^{*} \frac{4 R_{\mathrm{g}}}{\xi_{\mathrm{st}}+2 R_{\mathrm{g}}} \quad\left(\text { for } C>C^{*}\right)
$$

with $\xi_{\mathrm{st}}=2 R_{\mathrm{g}}\left(C^{*} / C\right)^{1 / 3}$ (eq 14b). From a mass conservation relationship for $C>C^{*}, C_{\mathrm{f}}\left(2 R_{\mathrm{g}}+\xi\right)+$ $C_{\mathrm{b}}\left(\xi_{\mathrm{st}}-\xi\right)=\left(C_{\mathrm{m}}+C\right)\left(2 R_{\mathrm{g}}+\xi_{\mathrm{st}}\right)$, the $\xi$ dependence of $C_{\mathrm{f}}$ in the forward regime is found to be

$$
C_{\mathrm{f}}=C_{\mathrm{m}}+C+C^{*}\left(\frac{4 R_{\mathrm{g}}}{\xi_{\mathrm{st}}+2 R_{\mathrm{g}}}\right)\left(\frac{\xi_{\mathrm{st}}-\xi}{\xi+2 R_{\mathrm{g}}}\right)
$$$$
\text { (for } C>C^{*} \text { ) }
$$

Utilizing eqs 14-17, we can explicitly conduct the integral in eq 13 with $v=1 / 2$ to calculate the osmotic free energy increment on fusion of the two $f$-mer aggregates, $\Delta G_{\mathrm{os}}$. The results are summarized as

$$
\begin{aligned}
& \frac{\Delta G_{\mathrm{os}}}{k_{\mathrm{B}} T}=\{9 \ln 2\}\left(\frac{C_{\mathrm{m}}}{C^{*}}\right)^{2}+9\left(\frac{C_{\mathrm{m}}}{C^{*}}\right)+\frac{9}{2} \\
& \text { for } C<C^{*} \\
& \frac{\Delta G_{\mathrm{os}}}{k_{\mathrm{B}} T}=\left\{9 \ln \left(1+\left(C^{*} / C\right)^{1 / 3}\right)\right\} \\
& \times\left(\frac{C_{\mathrm{m}}+C}{C^{*}}-\frac{2}{1+\left(C^{*} / C\right)^{1 / 3}}\right)^{2} \\
&+18\left(1-\frac{1}{1+\left(C^{*} / C\right)^{1 / 3}}\right) \\
& \times\left(\frac{C_{\mathrm{m}}+C}{C^{*}}-\frac{2}{1+\left(C^{*} / C\right)^{1 / 3}}\right) \\
&+6\left\{1-\left(\frac{1}{1+\left(C^{*} / C\right)^{1 / 3}}\right)^{2}\right\}
\end{aligned}
$$

In eqs 18 and $19, K_{\Pi}$ has been assumed to be unity for simplicity. In the absence of the matrix dPB chains $\left(C_{\mathrm{m}}=0\right)$, eq 18 reduces to the previously derived expression of $\Delta G_{\text {os }}$ for $C<C^{*}$.

Comparison with Data Under the assumption of the dominance of the equilibrium in the state of the $f$-mer aggregate, the molecular weight $M(t)$ of the hPB chains well as the overall and overlap concentrations $C(t)$ and $C^{*}(t)$ of this aggregate at the time $t$ are related to the residual $\mathrm{hB}$ monomer fraction $\phi(t)$ as

$$
\begin{aligned}
& M(t)=M(\infty)\{1-\phi(t)\} \\
& C(t)=C(\infty)\{1-\phi(t)\} \\
& C^{*}(t)=C^{*}(\infty)\left\{\frac{M(t)}{M(\infty)}\right\}^{1-3 v}=C^{*}(\infty)\{1-\phi(t)\}^{1-3 v} \\
& (v \cong 1 / 2 \text { for our hPB })
\end{aligned}
$$

Thus, the osmotic free energy increment $\Delta G_{\text {os }}$ deter- 
mined by these $C$ and $C^{*}$ (eqs 18 and 19) is dependent on $\phi(t)$. As noted from eq 11, this dependence leads, in principle, to a nonlinearity in the time evolution equation of $\phi(t)$. However, the $\phi(t)$ data shown in Figures 4-11 do not show a very large deviation from the single-exponential decay, suggesting that the second term in eq 11 (contribution of the $2 f$-mer aggregate to the time evolution of $\phi$ ) is not overwhelming the first term (contribution from the dissociated hPB chains). Thus, to the first order perturbation approximation, we may linearize eq 11 by replacing $C$ and $C^{*}$ included in eq 18 and 19 by those in the absence of the aggregate contribution (obtained from eq 11 with $B=0$ ),

$$
C^{\mathrm{o}}(t)=C(\infty)\{1-\exp (-t / \tau)\}
$$

and

$$
\begin{array}{r}
C^{0 *}(t)=C^{*}(\infty)\{1-\exp (-t / \tau)\}^{1-3 v} \\
(v \cong 1 / 2)
\end{array}
$$

Then, we can straightforwardly calculate $\phi(t)$ numerically if we know the values of the parameters $k_{\mathrm{p}} K_{\mathrm{d}}{ }^{1 / f}$ and $k_{\mathrm{p}}{ }^{\prime} / K_{\mathrm{d} 0}{ }^{\prime}$ included in $\tau$ and $B ; c f$. eq 12 . (Note that the other parameters appearing in eq $12, f(=4)$ and $[\mathrm{I}]_{0}$, are known.)

In Figures 8-11, the solid curves indicate the $\phi(t)$ calculated in this way for a single set of the parameters, $k_{\mathrm{p}} K_{\mathrm{d}}{ }^{1 / f}=0.19 \mathrm{~mol}^{-1 / 4} \mathrm{~cm}^{3 / 4} \mathrm{~min}^{-1}$ and $k_{\mathrm{p}}{ }^{\prime} /$ $K_{\mathrm{d} 0^{\prime}}=1.2 \times 10^{10} \mathrm{~mol}^{-2} \mathrm{~cm}^{6} \mathrm{~min}^{-1}$. The calculated curves are considerably close to the $\phi(t)$ data and reproduces the qualitative features, moderate slowing of the decay of $\log \phi(t)$ at long $t$, almost universal dependence of $\log \phi(t)$ on $t_{\mathrm{n}}=t\left\{[\mathrm{I}]_{0} / f\right\}^{1 / f}$ in the absence of matrix dPB (Figure 8), and the retarded decay in the presence of the matrix $\mathrm{dPB}$. These results strongly suggest that the osmotically affected propagation through the fused aggregates competes with the propagation through the dissociated chains.

In relation to this point, we should emphasize that the fusion-aided propagation mechanism vanishes at large $C(t)$ (or at large $C(t)+C_{\mathrm{m}}$ ) because of the enhanced osmotic barrier for fusion. Thus, the singleexponential decay of $\phi(t)$ due only to the propagation through the dissociated chains is recovered at sufficiently long $t$ where $C(t)$ (or $C(t)+C_{\mathrm{m}}$ ) is well above $C^{*}(t)$; $c f$. Figures 4-7 and 9-11. In other words, our result suggests that the pure contribution of the dissociated chains to the polymerization process is observed only at long $t$ and the (approximately) singleexponential decay at short $t$ seen in Figures 4-7 is not exclusively related to these chains.

\section{Contribution of nf-mer Aggregates to Propagation}

Here, we should emphasize that the model calculation presented above is based on the simplest assump- tion that the monodisperse $f$-mer aggregates are in equilibrium with the dissociated chains and the monodisperse $2 f$-mer aggregates. In the real solutions, the aggregation number $f$ has a distribution, ${ }^{15,16}$ and fused $n f$-mer aggregates with $n>2$ may be also formed. From this point of view, a very quantitative agreement cannot be expected between the above model and $\phi(t)$ data. However, the semi-quantitative agreement seen in Figures 8-11 strongly suggests that the fused aggregates (having a size $(n)$ distribution) contribute to the early-stage propagation: We can qualitatively confirm this point from the following argument.

We consider a situation that the most stable $f$-mer aggregates are in equilibrium with $n f$-mer aggregates with $n>2$ as well as the dissociated chains and $f$-mer aggregates and the equilibrium is predominantly in the $f$-mer aggregate state. For this case, it is easy to reformulate the reaction kinetics and modify eq 11 in a form:

$$
\begin{aligned}
\frac{\mathrm{d}\{\ln \phi(t)\}}{\mathrm{d} t}= & -\frac{1}{\tau}-B \exp \left(-\frac{\Delta G_{\mathrm{os}}(t)}{k_{\mathrm{B}} T}\right) \\
& -\sum_{n>2} B^{(n)} \exp \left(-\frac{\Delta G_{\mathrm{os}}^{(n)}(t)}{k_{\mathrm{B}} T}\right)
\end{aligned}
$$

with

$$
B^{(n)}=\left\{\frac{k_{\mathrm{p}}^{(n)}}{K_{\mathrm{d} 0}^{(n)}}\right\}\left\{\frac{[\mathrm{I}]_{0}}{f}\right\}^{n}
$$

The parameters $\tau$ and $B$ are given by eq $12,[\mathrm{I}]_{0}$ is the molar concentration of the initiator at the onset of propagation, and $k_{\mathrm{p}}^{(n)}$ and $K_{\mathrm{d} 0}^{(n)}$ represent the propagation rate constant and the non-osmotic part of the equilibrium constant for the $n f$-mer aggregate. $\Delta G_{\mathrm{os}}(t)$ and $\Delta G_{\mathrm{os}}^{(n)}(t)$, respectively, denote the osmotic penalties for fusion of the $f$-mer aggregates into $2 f$ mer and $n f$-mer aggregates.

Equation 22 suggests that the fused $2 f$-mer and $n f$ mer aggregates should have non-negligible contributions to the decay of $\phi(t)$ at short $t$ where the total polymer concentration remains sufficiently small and the osmotic penalties $\Delta G_{\mathrm{os}}(t)$ and $\Delta G_{\mathrm{os}}^{(n)}(t)$ are not significant. In contrast, at long $t$, the polymer concentration increases to enhance the osmotic penalties thereby suppressing the aggregate contributions and retarding the decay of $\phi(t)$. (At such long $t$, the propagation occurs only through the dissociated chains.) In the presence of the matrix $\mathrm{dPB}$, the osmotic penalties are enhanced even at short $t$ and the propagation is slower compared to that in the absence of dPB.

From these arguments, we may safely conclude the importance of the osmotic effect on the fused aggregates in the early-stage propagation even if the aggregates have a size distribution. In addition, the osmotic penalty should increase with increasing size $n$ of the fused aggregate (because a larger concentration gradi- 
ent is created on the fusion into larger aggregates) and the fusion-aided propagation would occur more easily for the $2 f$-mer aggregate than for the larger $n f$-mer aggregates with $n>2$, which probably resulted in the considerable success of the simple model explained in the previous section. (At this moment, it is difficult to quantitatively compare the prediction of eq 22 and the $\phi(t)$ data because this comparison requires us to estimate the values of many parameters $\left(B^{(n)}\right.$ with $\left.n>2\right)$. The comparison will be more easily made if the non-osmotic parts of the equilibrium constants $\left(K_{\mathrm{d} 0}^{(n)}\right.$ with $\left.n>2\right)$ are known from some independent experiments. These experiments and the resulting quantitative comparison are considered to be an important subject of future work.)

\section{Contribution of Decomposed Fragments of f-mer Aggregates to Propagation}

One may claim that the propagation could also occur through fragments (such as $f / 2$-mer aggregates) decomposed from the most stable $f$-mer aggregate because such fragments are less stable/more reactive compared to the $f$-mer aggregates. Our results do not rule out this possibility. However, the key observation presented in this paper is the deviation from the non-single exponential decay of $\phi(t)$ in the absence of the matrix $\mathrm{dPB}$ and the retardation of the decay in the presence of $\mathrm{dPB}$. We note that the propagation through the decomposed fragments should require no large-scale motion of the fragments, i.e., no significant increase in the local concentration of the polymeric components, because the monomers are much more mobile compared to these fragments and the molar concentration (not the mass concentration) of the monomer remains large even at long $t$ covered by our experiments. In other words, the monomer always exists in a vicinity of the decomposed fragment and the propagation occurs without an osmotic penalty as soon as the chemically active fragment is formed. (This feature is quite different from that for the propagation through fused aggregates: The fusion-aided propagation always requires an increase of the local concentration and is associated with the osmotic penalty.)

Thus, no significant osmotic effect is expected for the propagation through the decomposed fragments, as similar to the situation discussed earlier for the propagation through the dissociated chains. For this case, eq 22 is just modified as

$$
\begin{aligned}
\frac{\mathrm{d}\{\ln \phi(t)\}}{\mathrm{d} t}= & -\frac{1}{\tau^{\prime}}-B \exp \left(-\frac{\Delta G_{\mathrm{os}}(t)}{k_{\mathrm{B}} T}\right) \\
& -\sum_{n>2} B^{(n)} \exp \left(-\frac{\Delta G_{\mathrm{os}}^{(n)}(t)}{k_{\mathrm{B}} T}\right)
\end{aligned}
$$

where $\tau^{\prime}$ is a time-independent constant including the contribution from the decomposed fragments. (For example, if the $f / 2$-mer aggregate is the only fragment contributing to the propagation, $\tau^{\prime}$ is given by $\left[k_{\mathrm{p}, f / 2}\left\{K_{\mathrm{d}, f / 2}[\mathrm{I}]_{0} / f\right\}^{1 / 2}+\tau^{-1}\right]^{-1}$ with $k_{\mathrm{p}, f / 2}$ and $K_{\mathrm{d}, f / 2}$ being the propagation rate constant and equilibrium constant for the $f / 2$-mer aggregate.) Clearly, eq 24 gives the single-exponential decay of $\phi(t)$ in the absence of the last two terms, i.e., in the absence of the fusion-aided propagation. Thus, the propagation through the decomposed fragments of the $f$-mer aggregates may have actually occurred but this type of propagation is not responsible for the deviation from the single exponential decay of $\phi(t)$ in the absence of the matrix $\mathrm{dPB}$ and the retardation of the decay in the presence of $\mathrm{dPB}$.

\section{A Comment for Li-Li Exchange}

We have discussed the importance of the fusion of the most stable $f$-mer aggregates in the early-stage propagation. In relation to this point, we should note that the $\mathrm{Li}-\mathrm{Li}$ exchange corresponding to the fusion of the aggregates slows during the propagation process (Figure 12) but the propagation proceeds at a constant rate $(=-\{\mathrm{d} \ln \phi(t)\} / \mathrm{d} t)$ at long $t$ through the dissociated chains (Figures 4-11). Thus, a chain dissociating from a given aggregate seems to mostly return to this aggregate thereby allowing its $\mathrm{Li}$ (at the end) to recover the original chemical state. In other words, the single-chain dissociation process hardly contributes to the effective exchange of Li species. This situation, similar to that for a sticky Rouse chain, ${ }^{24}$ possibly reflects a fact that the original aggregate is the closest to the dissociated chain.

\section{CONCLUDING REMARKS}

We have utilized the ${ }^{1} \mathrm{H}$ NMR method to examine the kinetics in the polymerization (propagation) process of hPBLi in a nonpolar solvent, dBz. The hPBLi chains mostly form aggregates through $\mathrm{Li}$ at their ends. The residual monomer fraction $\phi(t)$ did not rigorously exhibit the single-exponential decay, $\phi(t)=$ $\exp (-t / \tau)$, expected from a molecular picture of the propagation only through the dissociated chains (and through fragments such as $f / 2$-mer aggregates decomposed from the most stable $f$-mer aggregates).

This deviation from the conventional behavior appears to be due to a competing propagation mechanism through the transiently fused aggregates: This fusion-aided propagation is osmotically suppressed on an increase of the hPB concentration (decrease of $\phi)$ and/or in the presence of a chemically inert dPB chains that behave as an osmotic tuner. The $\phi(t)$ data in the absence/presence of the $\mathrm{dPB}$ chains were semi- 
quantitatively described by a simple model considering the competition of the propagation processes through the fused aggregates and dissociated chains, with the latter mechanism vanishing in the late stage of propagation. This result suggests a non-negligible contribution of the fused aggregates to the living anionic polymerization kinetics. In this sense, the anionic polymerization is different from the other type of living polymerization (for example, living radical polymerization ${ }^{25}$ ) that includes no aggregation of the propagating chains.

\section{REFERENCES}

1. M. Morton, “Anionic Polymerization: Principles and Practice." Academic Press, New York, 1983.

2. M. Szwarc, Adv. Polym. Sci., 49, 1 (1983).

3. M. Szwarc and M. van Beylen, "Ionic Polymerization and Living Polymers," Chapman and Hall, New York, 1993.

4. H. L. Hsieh and R. P. Quirk, "Anionic Polymerization," Marcel Dekker, New York, 1996.

5. M. Morton, E. E. Bostick, R. A. Livigni, and L. J. Fetters, J. Polym. Sci. A, 1, 1735 (1963).

6. L. J. Fetters, J. Res. Natl. Bur. Std. Sect. A, 69, 159 (1965).

7. H. Hsieh, J Polym. Sci. A, 3, 153 (1965).

8. J. M. Alvarino, A. Bello, and G. M. Guzman, Eur. Polym. J., 8, 53 (1972).

9. R. N. Young, R. P. Quirk, and L. J. Fetters, Adv. Polym. Sci., 56, 1 (1984).

10. A. Z. Niu, J. Stellbrink, J. Allgaier, L. Willner, D. Richter, B. W. Koenig, M. Gondorf, S. Willbold, L. J. Fetters, and
R. P. May, Macromol. Symp., 215, 1 (2004).

11. L. J. Fetters, N. P. Balsara, J. S. Huang, H. S. Jeon, K. Almdal, and M. Y. Lin, Macromolecules, 28, 4996 (1995).

12. J. Stellbrink, L. Willner, O. Jucknischke, D. Richter, P. Lindner, L. J. Fetters, and J. S. Huang, Macromolecules, 31, 4189 (1998).

13. J. Stellbrink, L. Willner, D. Richter, P. Linder, L. J. Fetters, and J. S. Huang, Macromolecules, 32, 5321 (1999).

14. A. Z. Niu, J. Stellbrink, J. Allgaier, L. Willner, A. Radulescu, D. Richter, B. W. Koenig, R. P. May, and L. J. Fetters, J. Chem. Phys., 122, 134906 (2005).

15. Y. Matsuda, T. Sato, Y. Oishi, and H. Watanabe, J. Polym. Sci., Part B: Polym. Phys., 43, 1401 (2005).

16. Y. Oishi, H. Watanabe, T. Kanaya, H. Kaji, and F. Horii, Polym. J., 38, 277 (2006).

17. A. Frischknecht and S. T. Milner, J. Chem. Phys., 114, 1032 (2001).

18. Y. I. Estrin and T. S. Zyubina, Polym. Sci. Ser. A, 42, 1059 (2000).

19. H. Watanabe, O. Urakawa, H. Yamada, and M. L. Yao, Macromolecules, 29, 755 (1996).

20. L. J. Fetters, N. Hadjichristidis, J. S. Linder, and J. M. Mays, J. Phys. Chem. Ref. Data, 23, 619 (1994).

21. H. Yamakawa, "Modem Theory of Polymer Solutions," Harper \& Row, New York, 1971.

22. Y. Higo, N. Ueno, and I. Noda, Polym. J., 15, 367 (1983).

23. I. Noda, Y. Higo, N. Ueno, and T. Fujimoto, Macromolecules, 17, 1055 (1984).

24. M. Rubinstein and A. N. Semenov, Macromolecules, 31, 1386 (1998).

25. A. Goto and T. Fukuda, Prog. Polym. Sci., 29, 329 (2004). 